\title{
Early B cell changes predict autoimmunity following combination immune checkpoint blockade
}

\author{
Rituparna Das, ${ }^{1}$ Noffar Bar, ${ }^{1}$ Michelle Ferreira, ${ }^{1,2}$ Aaron M. Newman, ${ }^{3,4}$ Lin Zhang, ${ }^{1}$ Jithendra Kini Bailur, ${ }^{1}$ Antonella Bacchiocchi, \\ Harriet Kluger, ${ }^{1}$ Wei Wei, ${ }^{6}$ Ruth Halaban, ${ }^{5}$ Mario Sznol, ${ }^{1,7}$ Madhav V. Dhodapkar, ${ }^{1,7,8}$ and Kavita M. Dhodapkar ${ }^{2,7}$ \\ Department of Medicine, ${ }^{2}$ Department of Pediatrics, Yale University School of Medicine, New Haven, Connecticut, USA. ${ }^{3}$ Institute for Stem Cell Biology and Regenerative Medicine, and ${ }^{4}$ Department of \\ Biomedical Data Science, Stanford University, Stanford, California, USA. ${ }^{5}$ Department of Dermatology, ${ }^{6}$ Yale Center for Analytic Sciences, ${ }^{7}$ Yale Cancer Center, and ${ }^{8}$ Department of Immunobiology, Yale \\ University School of Medicine, New Haven, Connecticut, USA.
}

\begin{abstract}
Combination checkpoint blockade (CCB) targeting inhibitory CTLA4 and PD1 receptors holds promise for cancer therapy. Immune-related adverse events (IRAEs) remain a major obstacle for the optimal application of CCB in cancer. Here, we analyzed B cell changes in patients with melanoma following treatment with either anti-CTLA4 or anti-PD1, or in combination. CCB therapy led to changes in circulating B cells that were detectable after the first cycle of therapy and characterized by a decline in circulating $B$ cells and an increase in $C D 21^{10} B$ cells and plasmablasts. PD1 expression was higher in the CD21 $\mathrm{B}$ cells, and $B$ cell receptor sequencing of these cells demonstrated greater clonality and a higher frequency of clones compared with $\mathrm{CD} 21^{\mathrm{hi}}$ cells. CCB induced proliferation in the $\mathrm{CD} 21^{10}$ compartment, and single-cell RNA sequencing identified B cell activation in cells with genomic profiles of $\mathrm{CD} 21^{10} \mathrm{~B}$ cells in vivo. Increased clonality of circulating B cells following CCB occurred in some patients. Treatment-induced changes in B cells preceded and correlated with both the frequency and timing of IRAEs. Patients with early B cell changes experienced higher rates of grade 3 or higher IRAEs 6 months after CCB. Thus, early changes in B cells following CCB may identify patients who are at increased risk of IRAEs, and preemptive strategies targeting B cells may reduce toxicities in these patients.
\end{abstract}

\section{Introduction}

Combination checkpoint blockade (CCB) therapy with antiCTLA4 and anti-PD1 for the treatment of patients with melanoma leads to higher response rates and progression-free survival compared with treatment with either drug alone $(1,2)$. Unfortunately, CCB therapy is associated with a significant increase in immune-related adverse events (IRAEs) including treatmentrelated grade 3 or higher IRAEs in over half the patients, leading to discontinuation of therapy in approximately $40 \%$ of patients (2, 3). Development of IRAEs is therefore a major obstacle to the optimal application and evaluation of CCB therapy (3).

CTLA4 and PD1 have been implicated in the regulation of both B and T cell tolerance (4). Humans with heterozygous CTLA4 germline mutations develop B cell alterations and have an increased risk of autoimmunity (5). In prior studies, we showed that administration of anti-PD1 and anti-CTLA4 leads to distinct genomic signatures in $\mathrm{T}$ cells and myeloid cells compared with treatment using either drug alone (6), suggesting that combination therapy may be viewed as a unique treatment rather than as a combination of the 2 monotherapies. While some of the IRAEs are

Related Commentary: p. 577

Authorship note: R. Das and N. Bar are co-first authors.

Conflict of interest: The authors have declared that no conflict of interest exists.

Submitted: August 8, 2017; Accepted: November 7, 2017

Reference information: J Clin Invest. 2018;128(2):715-720.

https://doi.org/10.1172/JCI96798 thought to be $\mathrm{T}$ cell mediated, B cells have also been implicated (7-10). However, most studies monitoring immune responses following checkpoint blockade have focused on T cells, and systematic analyses of early changes in B cells and their correlation with autoimmunity are lacking. From a practical perspective, it would be highly desirable for patients at increased risk of developing autoimmunity to be identified before clinical toxicity and for their risk of autoimmunity to be reduced by preemptive intervention, without affecting clinical efficacy.

\section{Results and Discussion}

We analyzed changes in circulating B cells before and after the first cycle of therapy in 39 patients with advanced melanoma receiving immune checkpoint blockade (23 received combination therapy, 8 received anti-CTLA4, and 8 received anti-PD1; the patients' characteristics are provided in Supplemental Table 1; supplemental material available online with this article; https:// doi.org/10.1172/JCI96798DS1). Patients treated with CCB experienced a significant decrease in the number of circulating $\mathrm{B}$ cells (mean fold change, $0.7 ; P \leq 0.0001$ ) (Figure 1A), which we did not observe in patients treated with either anti-CTLA4 (mean fold change, $0.9 ; P=0.6$ ) or anti-PD1 (mean fold change, $1.1 ; P=0.13$ ) monotherapy. We also observed this difference when comparing absolute $\mathrm{B}$ cell counts before and after combination therapy $(P=$ 0.01; Supplemental Figure 1). Analysis of naive versus memory B cell subsets revealed no significant changes in any cohort (Supplemental Figure 2A). However, we observed a modest increase in the proportion of the class-switched memory cell subset after therapy 
A

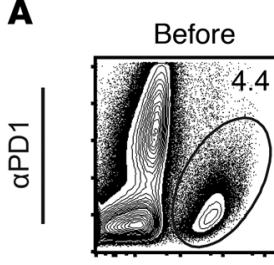

志
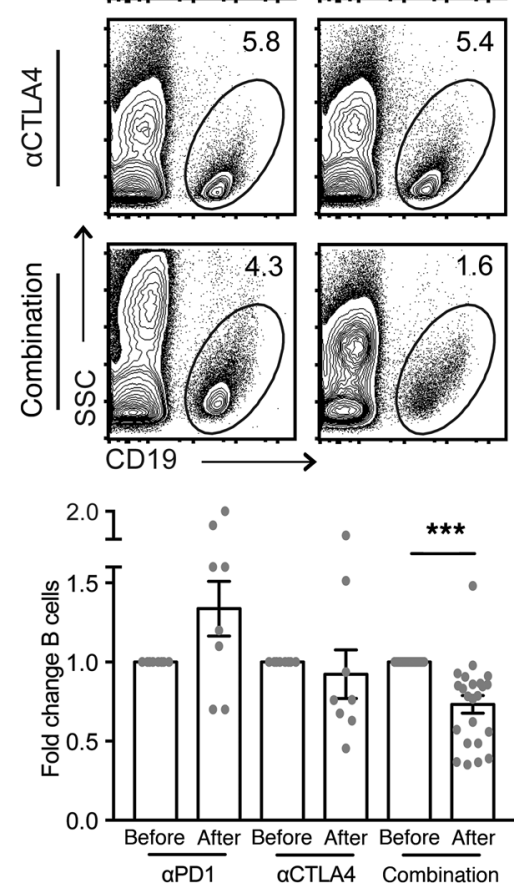

5.4
B

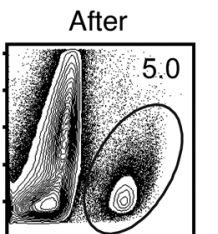

\section{志}
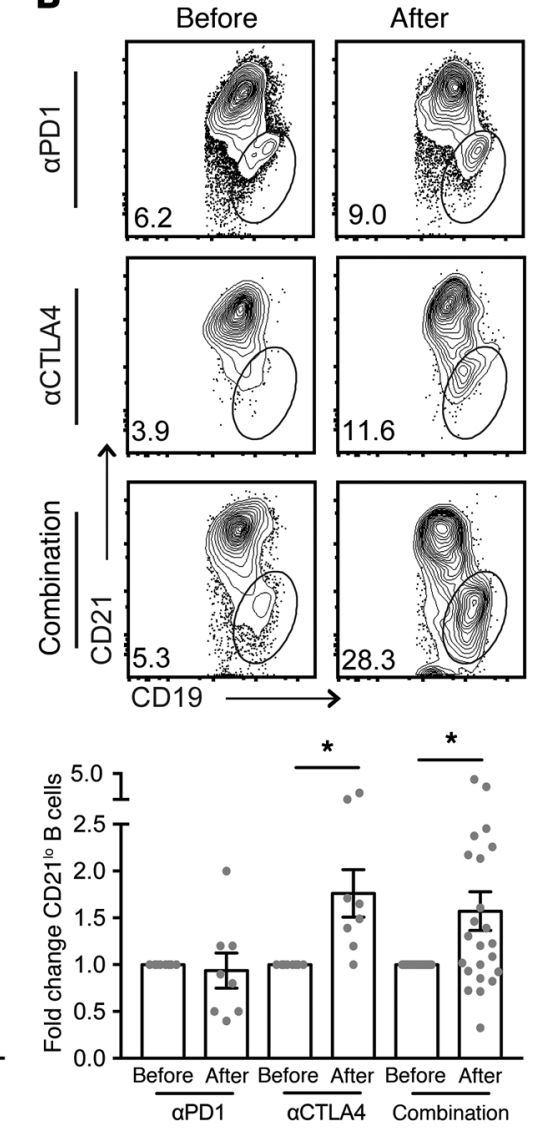

C
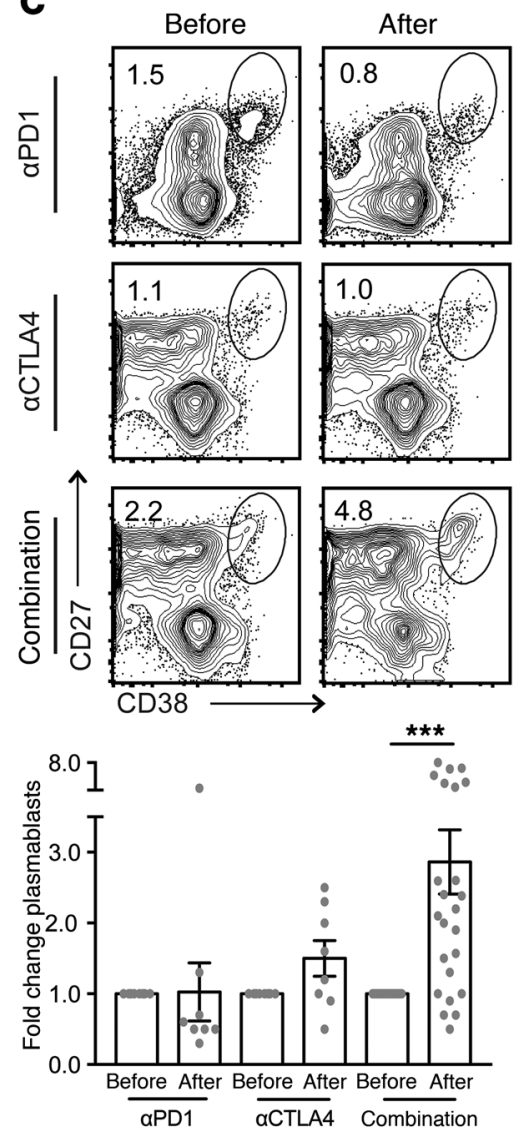

Figure 1. Distinct, early changes in circulating B cells following immune checkpoint therapy. Peripheral blood mononuclear cells (PBMCs), obtained from patients before and after the first cycle of therapy with either anti-PD1 ( $\alpha$ PD1, $n=8)$, anti-CTLA4 ( $\alpha$ CTLA4, $n=8)$, or concurrent administration of both anti-PD1 and anti-CTLA4 (Combination, $n=23$ ), were thawed, stained, and analyzed using flow cytometry. Shown are representative flow plots for all patients studied. Bar graphs indicate the fold change compared with before therapy. (A) Changes in circulating B cells are represented as the percentage of

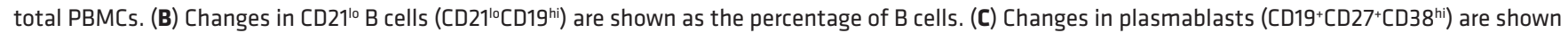
as the percentage of $B$ cells. All data represent the mean $\pm S E M .{ }^{*} P \leq 0.05$ and ${ }^{* * *} P<0.001$ by 2 -tailed Wilcoxon signed rank test.

in the combination therapy cohort $(P=0.0005$; Supplemental Figure $2 \mathrm{~B})$. Further analysis revealed an increase in the $\mathrm{CD} 21^{\mathrm{lo}} \mathrm{B}$ cell subset in patients treated with $\mathrm{CCB}$ (fold change, $1.6 ; P=0.01$ ) and with anti-CTLA4 alone (fold change, $1.8 ; P=0.02$ ), but not in the cohort treated with anti-PD1 alone (Figure 1B). CCB also led to a greater increase in plasmablasts compared with that seen in the monotherapy-treated cohorts (fold change, $2.9 ; P<0.0001$; Figure 1C). Plasma levels of CXCL13 were recently described as a marker of germinal center activation in humans (11). Given that the changes in B cells suggested germinal center activation, we examined CXCL13 levels in the plasma of patients before and after therapy. Combination therapy led to a greater increase in plasma CXCL13 levels compared with levels detected in the monotherapy cohorts $(P<0.0001$; Supplemental Figure 3). Thus, CCB therapy leads to distinct changes characterized by a decline in circulating B cells and an increase in $C D 21^{\text {lo }} \mathrm{B}$ cell subsets and plasmablasts.

$\mathrm{CD} 21^{\text {lo }} \mathrm{B}$ cells are a distinct $\mathrm{B}$ cell subset, however, their phenotype and functional properties differ in different settings (12, 13). Therefore, we evaluated these cells in detail in patients with melanoma. We found that equal numbers of naive and memory B cells were present at baseline in the $\mathrm{CD} 21^{10}$ compartment compared with the $C D 21^{\text {hi }} B$ cell subset, which contained pre- dominantly naive B cells (Supplemental Figure 4). CD21 $1^{\text {lo }}$ B cells showed a modest increase in memory $B$ cell numbers following $C C B$ therapy, whereas no changes were seen in $C D 21^{\text {hi }} B$ cell numbers (Supplemental Figure 4). B cells in the CD21 ${ }^{\text {lo }}$ subset also expressed higher levels of CD95 and lower levels of CD40 and lacked expression of the marrow- and lymphoid tissuehoming receptors CXCR4 and CXCR5 (Figure 2A). B cell receptor sequencing on flow-sorted $\mathrm{CD} 21^{\mathrm{hi}}$ and $\mathrm{CD} 21^{\mathrm{lo}} \mathrm{B}$ cells revealed that $\mathrm{CD} 21^{\text {lo }} \mathrm{B}$ cells had greater clonality (as measured by the $1 /$ normalized Shannon index), higher maximal clone frequency, and a higher frequency of somatic hypermutations (SHMs) (Figure 2, B-D). Taken together, these data show that $C D 21^{\text {lo }} \mathrm{B}$ cells are a distinct $B$ cell subset in melanoma patients and are more abundant following CCB in vivo.

Checkpoint blockade may impact B cell function by directly acting on B cells expressing the specific checkpoint or indirectly via effects on T cells or myeloid cells. Analysis of circulating B cells revealed that expression of PD1 was largely restricted to the $\mathrm{CD} 21^{1 \mathrm{lo}}$ subset (Figure $2 \mathrm{E}$ ). $\mathrm{CD} 21^{\text {1o }} \mathrm{B}$ cells are typically thought to be an anergic or exhausted B cell subset. However, following Ki67 staining, analysis of the proliferating B cell compartment revealed that the proliferating cells were restricted to the $\mathrm{CD} 21^{\mathrm{lo}} \mathrm{IgD}-\mathrm{CD} 27^{+}$ 
A

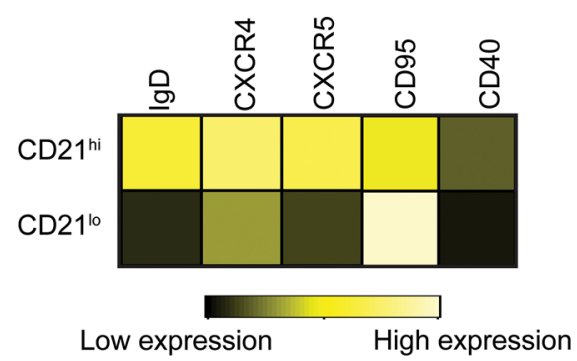

B

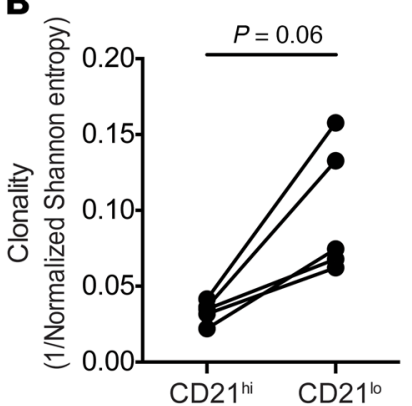

C

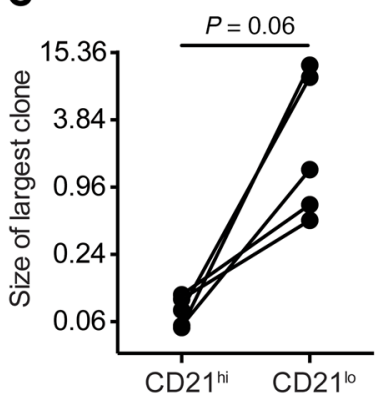

D

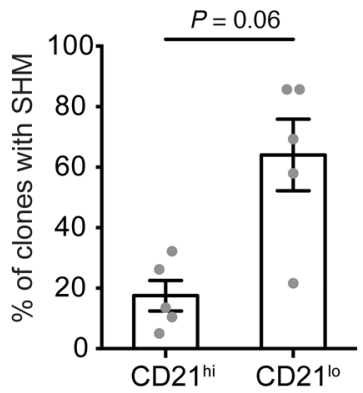

E

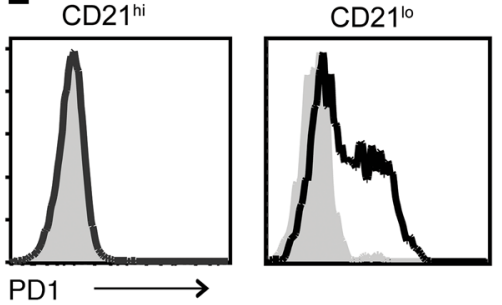

G

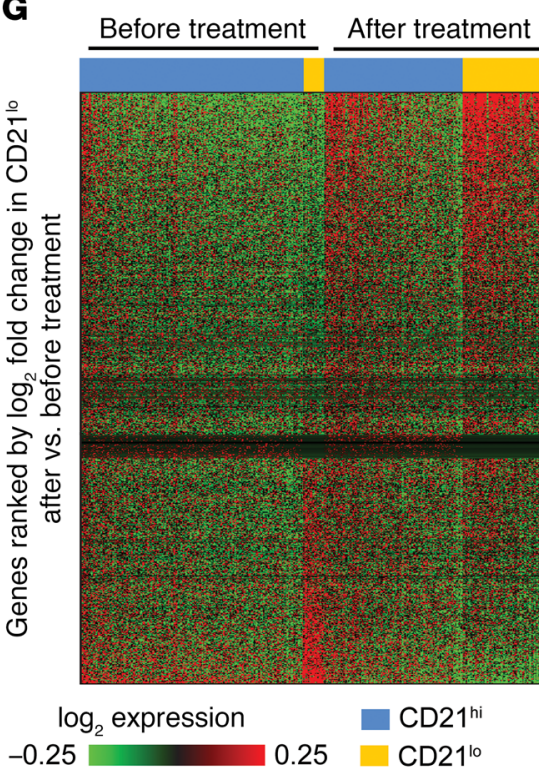

$\mathbf{F}$
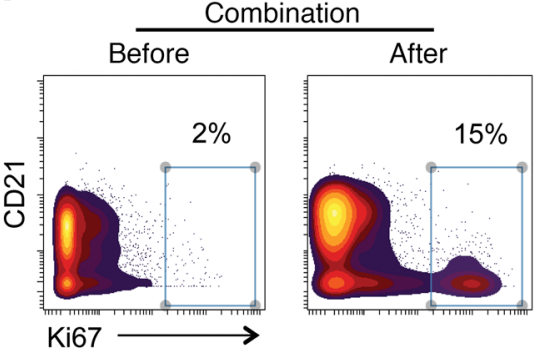

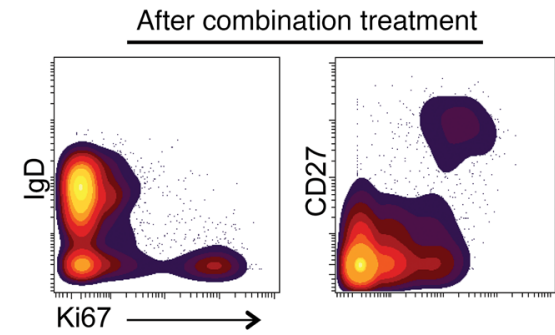

B cell IFN-y activation signaling

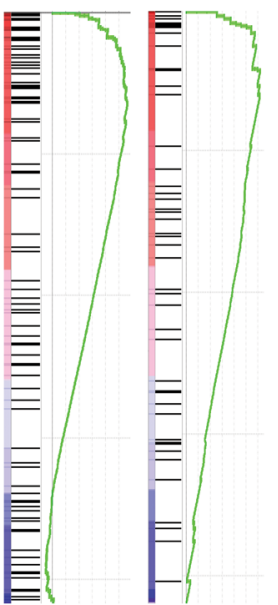

$Q=0.008 \quad Q=0.01$

$\mathrm{NES}=2.0 \quad \mathrm{NES}=1.9$

\begin{abstract}
Figure 2. Characteristics of $\mathrm{CD21}{ }^{10} \mathrm{~B}$ cells in patients receiving $\mathrm{CCB}$ therapy. (A) Mass cytometric (CyTOF) analysis of PBMCs from a patient with melanoma. Heatmap shows the expression of IgD, CXCR4, CXCR5, CD95, and CD40 on CD21 ${ }^{\text {hi }}$ and CD21 ${ }^{10} B$ cells. (B-D) CD21 $1^{\text {hi }}$ and CD21 $1^{10}$ cells were flow sorted and subjected to BCR sequencing $(n=5)$. Statistical data obtained via 2-tailed Wilcoxon signed rank test. (B) Clonality was defined as 1 /normalized Shannon entropy. (C) Size of the largest clone. (D) Percentage of $C D 21^{\mathrm{hi}}$ and $C D 21^{10} \mathrm{~B}$ cells with SHM. (E) Expression of PD1 (black line) on $\mathrm{CD} 21^{\text {hi }}$ and $\mathrm{CD} 21^{10} \mathrm{~B}$ cells. The isotype control antibody is shown as a gray histogram. (F) CyTOF analysis of proliferating B cells following CCB therapy. (G) B cells from a patient were flow sorted before and after CCB therapy, and single-cell RNA sequencing was performed. Heatmap shows genes that were differentially regulated in $C D 21^{10} B$ cells after therapy versus those prior to therapy for all $B$ cells analyzed. Panel on the right shows GSEA of pathways activated in post-treatment $C D 21^{10} B$ cells. NES, normalized enrichment score.
\end{abstract}

subset (Figure $2 \mathrm{~F}$ ). Since human $\mathrm{CD} 21^{\mathrm{lo}} \mathrm{B}$ cells have a distinct gene expression profile (12), we performed single-cell RNA sequencing on sort-purified $\mathrm{CD} 19^{+} \mathrm{B}$ cells from a patient before and after $\mathrm{CCB}$ and confirmed a post-therapy increase in B cells with a CD21 ${ }^{\text {lo }} \mathrm{B}$ cell genomic profile (Figure $2 \mathrm{G}$ ). Gene set enrichment analysis (GSEA) revealed a post-therapy increase in B cell activation as well as IFN- $\gamma$ signaling in the $\mathrm{CD} 21^{\text {lo }} \mathrm{B}$ cells (Figure $2 \mathrm{G}$ ). Together, these results identify $\mathrm{CD} 21^{\mathrm{lo}}$ memory $\mathrm{B}$ cells as a specific target of $\mathrm{CCB}$.

We sequenced the B cell receptor of bulk B cells (anti-PD1, $n=2$; anti-CTLA4, $n=5$; combination therapy, $n=10$ ) in order to gain further insights into checkpoint-related changes. Paired $\mathrm{B}$ cell receptor sequencing (BCR sequencing) analyses of bulk $\mathrm{B}$ cells before and after the first cycle of therapy revealed increased (>1.5-fold) B cell clonality in 2 of 10 patients given combination therapy, in 2 of 5 patients given anti-CTLA 4 monotherapy, and in 1 of 2 patients given anti-PD1 monotherapy (Figure 3A). However, we observed no significant changes in the maximal clone frequency before or after therapy (Supplemental Figure 5A). There were also no significant changes in $\mathrm{VH}$ family gene usage in samples before or after therapy, irrespective of changes in B cell clonality (Supplemental Figure 5B). An increase in BCR clonality did not correlate with the development of autoimmunity, but analysis of bulk B cells may have washed out the effects on specific subpopulations such as $\mathrm{CD} 21^{\mathrm{lo}} \mathrm{B}$ cells. Taken together, these data indicate that increased clonality of circulating B cells in response to checkpoint blockade occurs in some patients, but is not due to major expansion of a single clone.

Overall, grade 3 or higher IRAEs developed in 10 of 23 patients following combination therapy, in 1 of 8 patients following antiCTLA4 therapy, and in 1 of 8 patients following anti-PD1 therapy. The finding that CCB leads to higher rates of IRAEs as well as significant and distinct changes in B cells, including a decline in circu- 
A

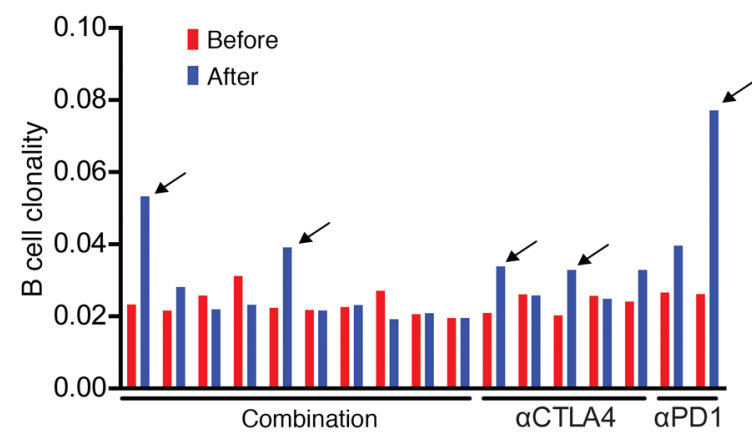

D

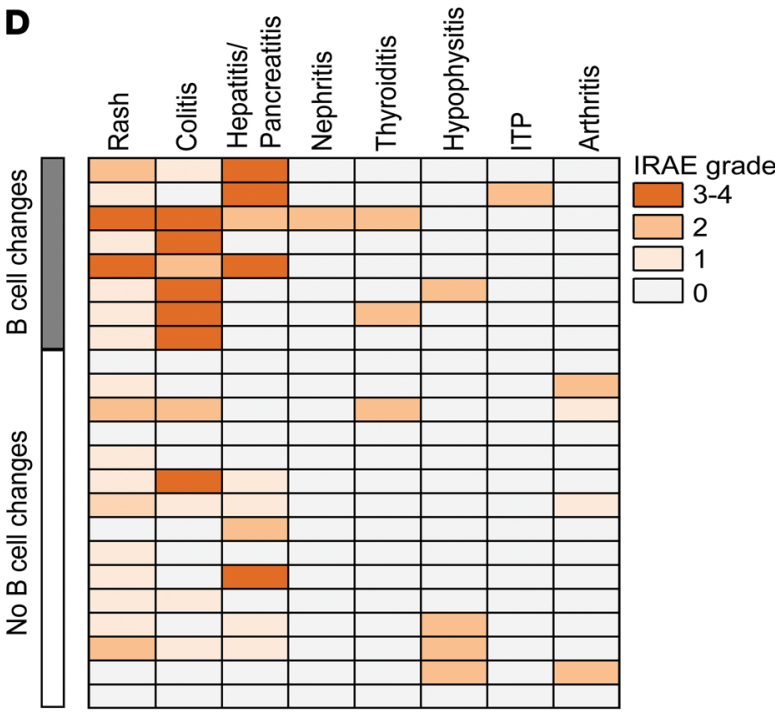

B

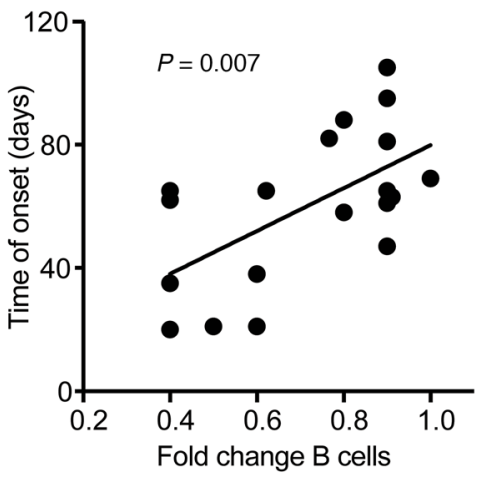

C

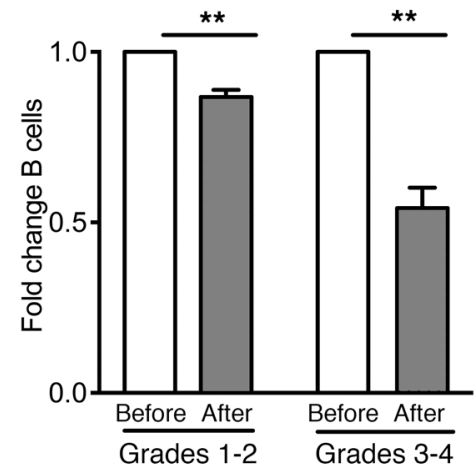

E

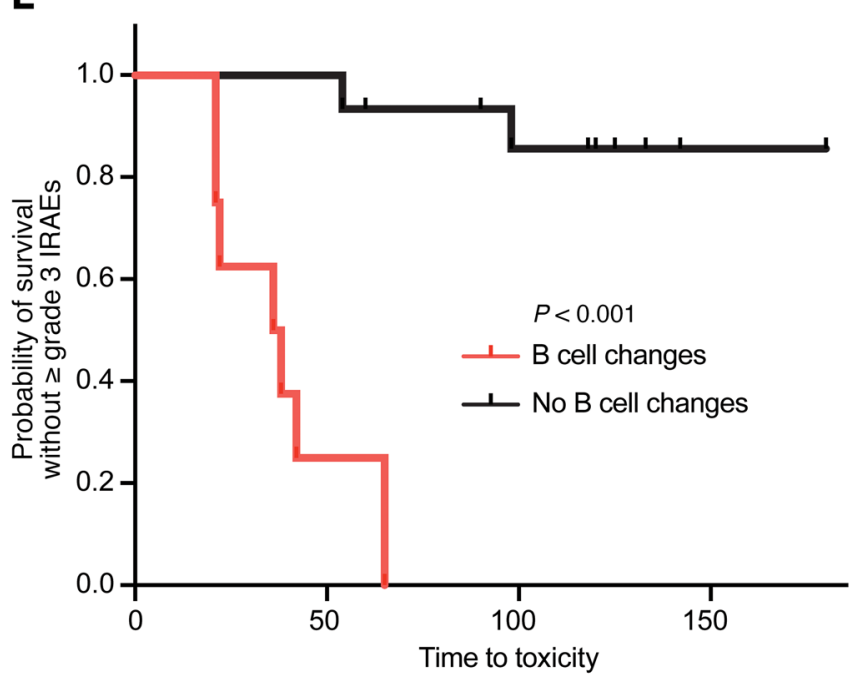

Figure 3. Combination immune checkpoint therapy correlates with development of IRAEs. (A) Bulk PBMCs obtained from patients before and after 1 cycle of therapy with either anti-PD1 $(n=2)$, anti-CTLA4 $(n=5)$, or combination therapy with both anti-PD1 and anti-CTLA4 $(n=10)$ were examined for their BCR clonality. Graph shows B cell clonality (1/normalized Shannon index) in individual patients before and after therapy. Arrows indicate patients with increased B cell clonality (>1.5-fold) after therapy. (B) Correlation between changes in circulating B cells and days to onset of a grade 2 IRAE in patients receiving CCB therapy. Statistical data obtained via linear regression analysis (C) Change in circulating B cells after CCB therapy in patients who developed a grade 1 or 2 IRAE $(n=10)$ as well as in those who developed a grade 3 or 4 IRAE $(n=10) .{ }^{*} P<0.01$, by 2 -tailed Wilcoxon signed rank test. (D) Type and grade for IRAEs seen in patients receiving CCB therapy $(n=23)$. ITP, immune thrombocytopenia. (E) Kaplan-Meier plot showing the probability of survival without development of a grade 3 or higher IRAE in patients with $(n=8)$ or without $(n=15)$ B cell changes (decline in B cells $<0.7$, with a $\geq 2$-fold increase in either $\mathrm{CD}_{2} 1^{10}$ cells or plasmablasts after therapy). Statistical data obtained via Fisher's exact test

lating $\mathrm{B}$ cells as well as an increase in $\mathrm{CD} 21^{\text {10 }} \mathrm{B}$ cells and plasmablasts, led us to evaluate the correlation between these changes and the development of autoimmunity. Interestingly, the severity of an early decline in B cell numbers after therapy directly correlated with the time to onset of toxicity (Figure 3B; $P=0.007$ ) as well as the grade of maximal toxicity (Figure $3 \mathrm{C}$ ). The ability to identify patients at increased risk for developing autoimmunity is critical for the application of CCB. We developed a parameter for $\mathrm{B}$ cell changes following combination therapy by integrating the decline in B cell numbers (fold change after therapy, $\leq 70 \%$ of baseline) with a 2-fold or greater increase in either $\mathrm{CD} 21^{1 \mathrm{o}} \mathrm{B}$ cells or plasmablasts. B cell changes preceded the clinical development of grade 3 or higher IRAEs by a median of 3 weeks (range, 0-10 weeks). Patients with treatment-induced B cell changes were more likely to develop multiorgan IRAEs than were those without such changes (Figure 3D). Overall, the cohort of patients with B cell changes had a greater risk of IRAEs, with rates of freedom from grade 3 or higher IRAEs at 6 months of $0 \%$ versus $87 \%(P<0.001$, Fisher's exact test; Figure 3E). Given the limitations of our sample size, we used bootstrap analyses to test the statistical robustness of our findings. Analysis of 100 bootstrap data sets revealed that the findings in the discovery data set could be replicated in all bootstrap data sets (Supplemental Figure 6). Changes in circulating T, NK, and myeloid cell numbers after therapy did not correlate with a risk of IRAEs (Supplemental Figure 7). Consistent with previous studies, CCB led to an increase in $\mathrm{Ki}^{+} 7^{+}$proliferating $\mathrm{CD} 4^{+}$and $\mathrm{CD} 8^{+} \mathrm{T}$ cells. However, the proportion of $\mathrm{Ki} 67^{+} \mathrm{T}$ cells did not correlate with the development of IRAEs (Supplemental Figures 7 and 8).

To our knowledge, these data provide the first detailed analysis of early changes in B cells following CCB therapy. IRAEs have emerged as a major challenge to the optimal application of this effective immunotherapy. While the changes in $\mathrm{T}$ cells following CCB therapy are well documented (6), we show that combination therapy also leads to distinct early changes in B cells, including a 
decline in circulating B cells, with an increase in the CD21 $1^{\text {lo }} \mathrm{B}$ cell subset and plasmablasts.

Our studies identify CD21 ${ }^{1 \mathrm{o}} \mathrm{B}$ cells as a distinct target of combination therapy. While these changes may be due to direct effects or indirect effects on other cell types, our findings are consistent with prior studies in humans with germline CTLA4 deficiency. Like our findings, these studies noted an overall decline in circulating B cells and a specific increase in circulating $C D 21^{\text {lo }} B$ cells (5). The finding that this subset of circulating B cells is specifically enriched for PD1 expression suggests that it may be essential to inhibit PD1 on these cells to fully unleash autoimmune manifestations. The mechanisms underlying the decline in circulating $\mathrm{B}$ cells following combination therapy is not known but may relate to enhanced tissue egress or plasma cell differentiation of CD21 ${ }^{\text {lo }}$ cells, as previously proposed to explain the low circulating B cell numbers in CTLA4-deficient humans (5).

The memory phenotype of $\mathrm{CD} 21^{1 \mathrm{o}} \mathrm{B}$ cells in melanoma seems to differ from the naive phenotype of these cells in lupus (14). $\mathrm{CD} 21^{\text {10 }} \mathrm{B}$ cells were recently described as new germinal center emigrants primed for plasma cell differentiation (15) and showed an overlap with tissue-homing, innate-like B cells (16). These properties position $\mathrm{CD} 21^{\mathrm{lo}} \mathrm{B}$ cells as being capable of rapid activation following checkpoint blockade and causing tissue injury. While $\mathrm{CD} 21^{\text {lo }} \mathrm{B}$ cells are thought to include many unresponsive or anergic clones (12), our data show that this is the precise subset of cells that undergoes proliferation in vivo following combination therapy.

The finding that early changes in B cell subsets is a strong predictor of IRAEs suggests that B cells may be important contributors to autoimmunity following combination therapy. Earlier studies have indeed demonstrated the presence of plasmablasts and $B$ cells in nonlymphoid tissue in the setting of CTLA4-deficient humans as well as in IRAEs following checkpoint blockade $(5,17)$. One limitation of our study is the small sample size, and further studies involving additional cohorts treated with combination therapy are needed.

Our data have several implications for the clinical optimization of immune checkpoint blockade. Major B cell contribution to IRAEs may provide the opportunity to separate mechanisms of autoimmunity from the well-established role of $\mathrm{T}$ cells in mediating tumor regression. B cell changes did not correlate with clinical response (partial response and complete response) to CCB in this cohort (Supplemental Figure 9). The strong correlation between early changes in B cells with the risk of subsequent IRAEs supports the need to monitor B cells as a simple tool to identify patients at risk for autoimmune toxicity. These findings provide the basis for the exploration of B cell-targeted therapies as a preemptive approach for at-risk individuals to improve their tolerance to CCB. Autoimmune complications of clinical checkpoint blockade ther- apies have proven difficult to replicate in preclinical models, and the underlying mechanisms may differ from mice to humans. Controlled clinical studies targeting B cells in patients undergoing $\mathrm{CCB}$ are needed to gain mechanistic insights into the contribution of B cells to CCB-induced IRAEs as well as antitumor effects.

\section{Methods}

Detailed methods are described in the Supplemental Methods.

Patients. Peripheral blood samples were obtained from patients before and after the first cycle of checkpoint therapy. The analysis of samples is detailed in the Supplemental Methods. Gene expression data were deposited in the NCBI's Gene Expression Omnibus (GEO) database (GEO GSE104600).

Statistics. Statistical significance was determined using a 2-tailed, nonparametric test for paired (Wilcoxon signed-rank test) or unpaired (Mann-Whitney $U$ test) samples. A $P$ value of less than 0.05 was considered significant. The time to toxicity was compared between the cohort with B cells changes and the cohort without B cell changes using the log-rank test, and the risk for IRAEs was determined using Fisher's exact test. Multiple comparisons correction was applied using the Bonferonni method. All data represent the mean \pm SEM.

Study approval. The study was approved by the IRB of Yale University. All subjects provided informed consent prior to their participation in the study.

\section{Author contributions}

$\mathrm{RD}$ and NB designed and performed experiments, analyzed data, and wrote the manuscript. MF, LZ, and JKB performed experiments and analyzed data. AM analyzed RNA-sequencing data. AB and RH helped to obtain clinical specimens and edited the manuscript. HK assisted in sample collection and response evaluation in some patients. WW performed biostatistical analysis. MS and MD analyzed data, designed experiments, and edited the manuscript. KD designed and supervised the study, analyzed data, and wrote the manuscript.

\section{Acknowledgments}

This work was supported in part by NIH grant CA197603 (to MVD) and the National Cancer Institute grant 1 P50 CA121974 (to RH, principal investigator). The authors acknowledge Guilin Wang (Yale Center for Genome Analysis) for help with single-cell RNA sequencing.

Address correspondence to: Kavita M. Dhodapkar or Madhav Dhodapkar, Yale School of Medicine, 333 Cedar Street, New Haven, Connecticut, USA. Phone: 203.785.4640; Email: kavita. dhodapkar@yale.edu (K.M. Dhodapkar). Phone: 203.785.4144; Email: madhav.dhodapkar@yale.edu (M. Dhodapkar).
1. Wolchok JD, et al. Nivolumab plus ipilimumab in advanced melanoma. $N$ Engl J Med. 2013;369(2):122-133.

2. Larkin J, et al. Combined nivolumab and ipilimumab or monotherapy in untreated melanoma. N Engl JMed. 2015;373(1):23-34.

3. Boutros C, et al. Safety profiles of anti-CTLA-4 and anti-PD-1 antibodies alone and in combination. Nat Rev Clin Oncol. 2016;13 (8):473-486.
4. Schildberg FA, Klein SR, Freeman GJ, Sharpe AH. Coinhibitory Pathways in the B7-CD28 LigandReceptor Family. Immunity. 2016;44 (5):955-972.

5. Kuehn HS, et al. Immune dysregulation in human subjects with heterozygous germline mutations in CTLA4. Science. 2014;345 (6204):1623-1627.

6. Das R, et al. Combination therapy with antiCTLA-4 and anti-PD-1 leads to distinct immunologic changes in vivo. J Immunol. 2015;194
(3):950-959.

7. Iwama S, De Remigis A, Callahan MK, Slovin SF, Wolchok JD, Caturegli P. Pituitary expression of CTLA-4 mediates hypophysitis secondary to administration of CTLA-4 blocking antibody. Sci Transl Med. 2014;6 (230):230ra45.

8. Naidoo J, et al. Autoimmune Bullous Skin Disorders with Immune Checkpoint Inhibitors Targeting PD-1 and PD-L1. Cancer Immunol Res. 
2016;4 (5):383-389.

9. Osorio JC, et al. Antibody-mediated thyroid dysfunction during T-cell checkpoint blockade in patients with non-small-cell lung cancer. Ann Oncol. 2017;28 (3):583-589.

10. Sznol M, et al. Endocrine-related adverse events associated with immune checkpoint blockade and expert insights on their management. Cancer Treat Rev. 2017;58:70-76.

11. Havenar-Daughton C, et al. CXCL13 is a plasma biomarker of germinal center activity. Proc Natl Acad Sci U S A . 2016;113 (10):2702-2707.
12. Isnardi I, et al. Complement receptor 2/CD21- human naive B cells contain mostly autoreactive unresponsive clones. Blood. 2010;115 (24):5026-5036.

13. Thorarinsdottir K, et al. CD21 (-/low) B cells in human blood are memory cells. Clin Exp Immunol. 2016;185 (2):252-262.

14. Tipton CM, et al. Diversity, cellular origin and autoreactivity of antibody-secreting cell population expansions in acute systemic lupus erythematosus. Nat Immunol. 2015;16 (7):755-765.

15. Lau D, et al. Low CD21 expression defines a population of recent germinal center graduates primed for plasma cell differentiation. Sci Immunol. 2017;2(7): eaai8153.

16. Rakhmanov M, Gutenberger S, Keller B, Schlesier M, Peter HH, Warnatz K. CD21low B cells in common variable immunodeficiency do not show defects in receptor editing, but resemble tissue-like memory B cells. Blood. 2010;116 (18):3682-3683.

17. Beck KE, et al. Enterocolitis in patients with cancer after antibody blockade of cytotoxic T-lymphocyte-associated antigen 4. J Clin Oncol. 2006;24 (15):2283-2289. 